\title{
Breast cancer therapy: a mini review
}

\begin{abstract}
Breast cancer is a prevailing disease worldwide that requires effective and rational therapy. For this purpose the use of various treatment modalities should be optimized according to the stage of disease and the risk: benefit ratio of the therapeutic agents employed in the patients. Although the therapies involved provide reduction in morbidity and mortality rates, monitoring is required to combat the resulting adverse drug reactions. This review would be helpful for healthcare professionals to address the multi factorial disease in accordance with its occurrence thereby providing rationalized therapy to the patients.
\end{abstract}

Keywords: breast cancer, chemotherapy, endocrine therapy, adjuvant therapy
Volume I Issue 2 - 2017

\author{
Fakhsheena Anjum,' Nighat Razvi,' MAli \\ Masood' \\ 'Dow University of Health Sciences, Pakistan \\ ${ }^{2}$ Nazeer Hussain University, Pakistan
}

\begin{abstract}
Correspondence: Fakhsheena Anjum, Dow College of Pharmacy, Dow University of Health Sciences, Karachi, Pakistan, Tel 0334-3355750,Email fakhsheena.anjum@duhs.edu.pk
\end{abstract}

Received: March II, 2017 | Published: May 16, 2017

\section{Introduction}

Breast cancer has increased universally ${ }^{1,2}$ and is considered as the second chief mortality cause in women. Among top five cancers in Americans, it is the third fatal cancer ${ }^{3}$ while it is also the most prevalent cancer in Asians. ${ }^{4}$ Since breast cancer is a very diverse ailment ${ }^{5}$ it has several indicators ${ }^{6}$ associated to typical features of tissues, therapeutic evaluation and reaction to therapy. ${ }^{7}$ Improvements in the diagnosis and management of breast cancer have yielded drop in mortality frequency, but it varies widely between diverse geographic areas. ${ }^{8,9}$ For early and locally advanced breast carcinoma, the intention of treatment is cure while for metastasis, it is improvement in clinical presentation of disease and quality of life. Various approaches are employed for breast cancer management like surgery, radiation treatment, endocrine treatment and chemotherapy. ${ }^{10}$ The management of breast cancer is discussed as follows since the disease requires proper monitoring and optimized treatment schedules in the patients. The incidence and risk factors of breast cancer vary geographically; also patients respond to the provided treatment differently, therefore, rationalized therapy in individual cases according to the stage of the disease is essential.

\section{General treatment recommendations according to breast cancer staging}

The staging of breast cancer is one of the most consistent prognostic signs that provide valuable confirmation about the current status of cancer identification and its therapy. The staging of breast cancer involves tumor size (T 1-4), association of lymph nodes (N 1-3) and existence of distant metastases (M 0-1). In stage 0, lumpectomy alone can be employed for Ductal Carcinoma in Situ $(<0.5 \mathrm{~cm}$ diameter) but for larger lesions, it is used with adjuvant radiation therapy. Extensive Ductal Carcinoma in Situ (i.e. involving two or more quadrants of the breast) requires mastectomy; adjuvant Tamoxifen therapy is considered in all patients. ${ }^{11}$

According to existing practice for Stage I and II, after considering axillary lymph node status, lumpectomy and radiation therapy are used in patients with stage I or II breast cancer; this has displayed equivalent effects for total mastectomy and for lumpectomy followed by radiation therapy with 5 and 8 year disease-free and overall survival rates. ${ }^{12}$ Due to surgical margins ensuing to elimination of a breast mass and definite connective tissue diseases and multi-centric disease, the patients with former radiation therapy to the breast or chest wall should go through mastectomy. ${ }^{13}$ A thorough axillary segmentation should be employed for an obvious axillary lymphadenopathy and patients having a primary cancer (T1 or T2) without involving axillary lymph nodes should undergo sentinel lymph node biopsy. For early breast cancer, systemic chemotherapy is used with radiotherapy. Large tumors $(>1 \mathrm{~cm})$ and node positive breast cancer are treated with chemotherapy. ${ }^{13}$ Adjuvant endocrine therapy is followed by hormone receptor status evaluation.

The stage IIIa and IIIc cancers are locally advanced which may be resectable and non resectable..$^{13}$ A modified radical mastectomy and adjuvant chemotherapy along with radiation therapy are employed to manage the resectable type; neo-adjuvant chemotherapy may be used in some patients in order to shrink the size of the primary tumor. ${ }^{14}$ Adjuvant endocrine therapy is followed by hormone receptor status evaluation. In the stages IIIb, IIIc and inflammatory breast carcinoma, multimodal approach is required to accomplish cure. Initially neoadjuvant chemotherapy is used and upon positive response, a major modified mastectomy tailed by radiation therapy to the chest wall and regional lymphatics may be employed. ${ }^{13}$ For some patients, chemotherapy after breast-conserving surgery may be advised. ${ }^{14}$ Adjuvant endocrine therapy is followed by hormone receptor status evaluation.

The stage IV of breast cancer has no cure and the treatment is focused on increased survival rates and quality of life improvement. The endocrine therapies are often suggested as first line management for the $\mathrm{ER}+$ or $\mathrm{PR}+$ cancers with only bone or soft tissue metastases, or with limited and asymptomatic visceral metastases. ${ }^{13}$ For the hormone-refractory cancers, ER- or PR- cancers, or symptomatic visceral metastases, systemic chemotherapy is the most appropriate. ${ }^{15}$ To control symptoms, palliation is essential and to evade bone fractures, bisphosphonates can be used for bone metastasis.

\section{Breast cancer therapy by class}

Treatment plan for breast carcinoma can be outlined by organized screening and identification of the ailment. ${ }^{16}$ The choice of therapy for breast carcinoma must outweigh benefits over risks; ${ }^{17}$ the dose, dosing plan and response of the therapeutic agents used for treatment should be monitored through regular follow ups. The therapeutic agents used 
for breast cancer treatment cause adverse drug reactions in addition to their therapeutic outcomes ${ }^{18}$ and such adverse reactions discourage patient adherence to the therapy; hence, the pre- and post- treatments to cope such indications must be in line with the standard treatment recommendations.

Various classes of therapeutic agents are employed for breast cancer treatment:

a. Alkylating agent: cyclophosphamide (nitrogen mustard)

b. Anti-metabolite: methotrexate (folic acid analogue), 5-fluorouracil \& capacitabine (pyramidine analogues)

c. Natural product: vinorelbine (vinca alkaloid), paclitaxel (taxane), doxorubicin (antibiotic)

d. Hormone and antagonist: tamoxifen (anti estrogen), letrozole \& anastrazole (aromatase inhibitors)

e. Miscellaneous: trastuzumab (monoclonal antibody), lapatinib (Protein tyrosine kinase inhibitor)

\section{Endocrine treatment}

Tamoxifen is a selective estrogen receptor modulator (SERM) ${ }^{19}$ that binds to Estrogen Receptor and has mixed agonist and antagonist features i.e its principal mechanism of action is facilitated by its binding to the estrogen receptor and inhibition of the proliferative activities of estrogen on mammary epithelium; ${ }^{20}$ Tamoxifen $20 \mathrm{mg}$ tablets are established as gold standard for breast cancer therapy. ${ }^{21}$ Reports have shown that with use of Tamoxifen, irrespective of menopausal or lymph node status, risk of ER+ breast cancer recurrence is reduced to $50 \%$ and also there is about $28 \%$ decrease in morbidity rates. ${ }^{22}$ On the contrary, it has been found that the endocrine treatments like Tamoxifen itself might intensify the xenoestrogens agonistic effects on transmuted Estrogen Receptors that are linked to drug resistance and refractoriness. ${ }^{23}$

Aromatase is the chief estrogen source in post menopausal females. As a substitute to Tamoxifen in post menopausal women, (especially in ER+breast cancer), third generation aromatase inhibitors i.e. letrozole, anastrozole and exemestane, are generally used. ${ }^{24}$ These agents are non steroidal that reversibly inhibit aromatase enzyme which transforms androstenedione into esterone and testosterone to estrogen. The drugs have exhibited enhanced positive results in the post menopausal females ${ }^{25,26}$ and they also have a better body tolerance than preceding hormonal treatments. ${ }^{27}$

\section{Chemotherapeutic agents}

Some of the most common regimens employed in breast cancer are discussed as follows.

Cyclophosphamide averts the DNA replication and cell division and is employed for the treating breast cancer metastasis. This pro drug transforms into active form via hepatic intracellular enzymes to active metabolites (i.e. 4 hydroxy cyclophosphamide, aldophosphamide, acrolein and phosphor amide mustard). ${ }^{27}$ The drug has been used generally as an adjuvant therapy in combination regime of CMF or with an anthracycline for treatment of breast cancer. ${ }^{26}$

Platinum compounds like Carboplatin and cisplatin are employed against several cancers and are employed as monotherapy or in combination regimen to treat breast cancer. ${ }^{27}$ The influence of platinum compounds on DNA conformation and stability has been studied and a number of platinum-DNA adducts have been known in vivo and in vitro. Earliest investigations have quantified the influence of these dissimilar lesions on replication of DNA, their ability to bring in mutations and their susceptibility to DNA repair procedures. Further damage to DNA may be generated by platinum (IV) compounds, possibly through their decline by the cell to platinum (II) compounds. ${ }^{28}$ About $20-35 \%$ patients of Metastatic Breast Cancer under mono therapy had been found responding to carboplatin therapy. ${ }^{29,30}$ Gemcitabine and Taxanes are the drugs that are used generally in combination with Platinum compounds. ${ }^{31,32}$

Paclitaxel and Docetaxel are the most commonly employed taxanes that cause mitotic arrest by stabilizing cellular microtubule elements. These agents have been used either as mono therapy or in combination schedule. ${ }^{33}$ A weekly management schedule of these agents has been reported to be well tolerated with little toxicity in breast cancer. ${ }^{34,35}$ Anthracyclines like Doxorubicin and Epirubicin (anthracyclines) are broadly used in the combination regimen (i.e. FAC, AC, TAC) to treat breast cancer. Several mechanisms have been suggested for elucidating the cytostatic and cytotoxic activities of anthracyclines i.e. these comprise of free radical development, lipid peroxidation, and direct effects of membrane. Among all the mechanisms, the best described are the interactions with the DNA itself or DNA-topoisomerase II complex through intercalation or covalent bonding and base amendments, which cause instabilities in DNA replication and transcription, and then the initiation of repair of DNA or apoptotic cell death. ${ }^{36}$

Despite of the toxicities, the multi drug combination regimens have proven efficacy for breast cancer. ${ }^{27}$ Capecitabine is an oral prodrug of fluoropyrimidine that transforms into 5-FU by thymidine phosphorylase enzyme rendering similar effects as those of 5-FU upon infusion. It is has been used next to use of taxanes for treatment of progressing Metastatic Breast Cancer. ${ }^{37}$ Gemcitabine (or difluoro deoxy cytidine) is a pyrimidine nucleotide that blocks RNA synthesis and DNA replication and is applied for treating several types of cancers i.e. cancer of lung, bladder, breast, etc. Gemcitabine is well tolerated upon weekly IV injection. ${ }^{27}$ Vinorelbine binds to the tubulin hence disrupting mitosis metaphase. This drug has exhibited promising results in advanced breast cancer as reported in various researches. ${ }^{38-40}$

\section{Monoclonal antibodies}

Trastuzumab is a biologically active, humanized monoclonal antibody which has improved survival rates for HER2/neu positive breast cancer patients; ${ }^{41,42}$ it works against the extracellular domain IV of HER $2 .{ }^{43}$ This monoclonal antibody is clinically safe and effective in regime every 3 weeks ${ }^{44}$ and is also effective in combination with paclitaxel, gemcitabine, vinorelbine or carboplatin. ${ }^{45,46}$

\section{Protein tyrosine kinase inhibitor}

Lapatinib is an orally active, reversible blocker of the EGFR and HER2 tyrosine kinase; its activity appears to be mainly due to HER $2 .{ }^{47}$ It has displayed induction of apoptosis of HER2-positive breast cancer cells as monotherpay, ${ }^{48}$ and re-established tamoxifen sensitivity to the resistant breast cancer cells. ${ }^{49}$ Lapatinib was established for use in combination with capecitabine when HER2-positive breast cancer exhibited progression over the use of trastuzumab; it is also used officially for HER2-positive metastatic breast cancer in combination with letrozole as first-line treatment. Lapatinib and chemotherapy combination accomplished $22 \%$ response rate and $27 \%$ clinical 
value rate ${ }^{50}$ and as monotherapy, it has displayed $12.4 \%$ to $25 \%$ clinical value rates in those patients who were earlier treated with trastuzumab; yet in some cases, limited resistance to lapatinib had also been detected. ${ }^{51,52}$

\section{Summary description for breast cancer therapy}

To reduce recurrence rate and augment survival rate, adjuvant therapy should be managed in all breast cancer patients. Aromatase Inhibitors must be employed as adjuvant therapy due to their efficacy instead of Tamoxifen for hormone receptor positive breast cancer in post menopausal women. Chemotherapy for 3-6 months should be given to premenopausal women and also to women having hormone receptor negative breast cancer. Efficacy of Anthracycline regimens as chemotherapeutic agents is proven for breast cancer and particularly in high risk patients, taxanes are used. For patients at high risk with HER2/neu positive breast cancer or with lymph node negative disease, chemotherapy plus trastuzumab is advised. ${ }^{27}$

For incurable Metastatic Breast Carcinoma, palliation is the treatment goal. Without widespread visceral involvement, for hormone receptor positive cancer, endocrine therapy is advised. Substitutes for anti hormones can be specified if first line or second line treatments fail. For widespread visceral involvement, advancement in disease on anti-hormonal treatment or for hormone receptor negative breast cancer, anthracyclines and taxanes can be directed. It has been recommended that mono-therapy in order can be just as effective for this stage since relative toxicities due to combination regimen require prompt resolution. The risk: benefit ratio of the therapies must be considered before considering many subsequent-line therapies for metastatic breast cancer if former chemotherapies fail. ${ }^{27}$

\section{Acknowledgements}

None.

\section{Conflict of interest}

The author declares no conflict of interest.

\section{References}

1. Hortobagyi GN, de la Garza Salazar J, Pritchard K, et al. The global breast cancer burden: variations in epidemiology and survival. Clin Breast Cancer. 2005;6(5):391-401.

2. Héry C, Ferlay J, Boniol M, et al. Changes in breast cancer incidence and mortality in middle-aged and elderly women in 28 countries with Caucasian majority populations. Ann Oncol. 2008;19(5):1009-1018.

3. Jemal A, Siegel R, Ward E, et al. Cancer statistics. CA: Cancer J Clinicians. 2008;58(2):71--96.

4. Kim H, Choi DH. Distribution of BRCA1 and BRCA2 mutations in asian patients with breast cancer. J Breast Cancer. 2013;16(4):357-365.

5. Polyak K. Breast cancer: origins and evolution. $J$ Clin Invest. 2007;117(11):3155-3163.

6. Qadir MI, Malik SA. Plasma lipid profile in gynecologic cancers. Eur J Gynaecol Oncol. 2007;29(2):158-161.

7. Weigelt B, Geyer FC, Reis Filho JS. Histological types of breast cancer: how special are they? Mol Oncol. 2010;4(3):192-208.
8. Sant M, Francisci S, Capocaccia R, et al. Time trends of breast cancer survival in Europe in relation to incidence and mortality. Int $J$ Cancer. 2006;119(10):2417-2422.

9. Autier P, Boniol M, La Vecchia C, et al. Disparities in breast cancer mortality trends between 30 European countries: retrospective trend analysis of WHO mortality database. BMJ. 2010;341:c3620.

10. Lukaszewicz K, Wtorek J, Bujnowski A, et al. Monitoring of breast tissue thermo-ablation by means of impedance measurements. J Physics: Conference Series, IOP Publishing; 2010. p. 1-4.

11. Fisher B, Costantino JP, Wickerham DL, et al. Tamoxifen for prevention of breast cancer: report of the National Surgical Adjuvant Breast and Bowel Project P-1 Study. J Natl Cancer Inst. 1998;90(18):1371-1388.

12. Arriagada R, Lê MG, Rochard F, et al. Conservative treatment versus mastectomy in early breast cancer: patterns of failure with 15 years of follow-up data. institut gustave-roussy breast cancer group. J Clin Oncol. 1996;14(5):1558-1564.

13. Singletary SE, Robb GL, Hortobagyi GN. Advanced therapy of breast disease. PMPH-USA; 2001.

14. Chen AM, Meric Bernstam F, Hunt KK, et al. Breast conservation after neo adjuvant chemotherapy: the MD Anderson cancer center experience. $J$ Clin Oncol. 2004;22(12):2303-2312.

15. Carlson RW, Allred DC, Anderson BO, et al. Breast cancer. J National Comprehensive Cancer Network. 2009;7(2):122-192.

16. Geay JF. Physiopathology, diagnosis and treatment of breast cancer. Soins. 2013;(776):25-29.

17. Maughan KL, Lutterbie MA, Ham PS. Treatment of breast cancer. Am Fam Physician. 2010;81(11):1339-1346.

18. Jose J, Rao PG. Pattern of adverse drug reactions notified by spontaneous reporting in an Indian tertiary care teaching hospital. Pharmacol Res. 2006;54(3):226-233.

19. Chen WY, Colditz GA. Risk factors and hormone-receptor status: epidemiology, risk-prediction models and treatment implications for breast cancer. Nat Clin Pract Oncol. 2007;4(7):415-423.

20. Fugh Berman A, Epstein S. Tamoxifen: disease prevention or disease substitution? Lancet. 1992;340(8828):1143-1145.

21. Riggs BL, Hartmann LC. Selective estrogen-receptor modulatorsmechanisms of action and application to clinical practice. $N$ Engl $J$ Med. 2003;348(7):618-629.

22. Group EBCTC. Poly chemotherapy for early breast cancer: an overview of the randomized trials. Lancet. 1998;352(9132):930-942.

23. Hess Wilson JK, Boldison J, Weaver KE, et al. Xenoestrogen action in breast cancer: impact on ER-dependent transcription and mitogenesis. Breast Cancer Res Treat. 2006;96(3):279-292.

24. Bonneterre J, Buzdar A, Nabholtz JM, et al. Anastrozole is superior to tamoxifen as first $\square$ line therapy in hormone receptor positive advanced breast carcinoma. Cancer. 2001;92(9):2247-2258.

25. Nabholtz JM, Falkson C, Campos D, et al. Docetaxel and doxorubicin compared with doxorubicin and cyclophosphamide as first-line chemotherapy for metastatic breast cancer: results of a randomized, multicenter, phase III trial. J Clin Oncol. 2003;21(6):968-975.

26. Mouridsen HT, Palshof T, Brahm M, et al. Evaluation of single-drug versus multiple-drug chemotherapy in the treatment of advanced breast cancer. Cancer Treat Rep. 1976;61(1):47-50. 
27. Waldman SA, Terzic A. Pharmacology and therapeutics: principles to practice. Canada: Elsevier; 2009. 1536 p.

28. Johnson NP, Butour JL, Villani G, et al. Metal Antitumor Compounds: The Mechanism of Action of Platinum Complexes. In: Baulieu E, editors. Ruthenium and Other Non-Platinum Metal Complexes in Cancer Chemotherapy. Progress in Clinical Biochem \&d Med, Springer; 1989. p. 1-24.

29. Martín M1, Díaz-Rubio E, Casado A, et al. Carboplatin: an active drug in metastatic breast cancer. J Clin Oncol. 1992;10(3):433-437.

30. O Brien ME, Talbot DC, Smith IE. Carboplatin in the treatment of advanced breast cancer: a phase II study using a pharmacokinetically guided dose schedule. J Clin Oncol. 1993;11(11):2112-2117.

31. Perez EA, Suman VJ, Rowland KM, et al. Two concurrent phase II trials of paclitaxel/carboplatin/trastuzumab (weekly or every-3-week schedule) as first-line therapy in women with HER2-overexpressing metastatic breas cancer: NCCTG study 983252. Clin Breast Cancer. 2005;6(5):425-432.

32. Burch PA, Mailliard JA, Hillman DW, et al. Phase II study of gemcitabine plus cisplatin in patients with metastatic breast cancer: a North central cancer treatment group trial. Am J Clin Oncol. 2005;28(2):195-200.

33. Sparano JA. Taxanes for breast cancer: an evidence-based review of randomized phase II and phase III trials. Clin Breast Cancer. 2000;1(1):3240 .

34. Burstein HJ, Manola J, Younger J, et al. Docetaxel administered on a weekly basis for metastatic breast cancer. J Clin Oncol. 2000;18(6):12121219.

35. Eniu A, Palmieri FM, Perez EA. Weekly administration of docetaxel and paclitaxel in metastatic or advanced breast cancer. Oncologist. 2005;10(9):665-685.

36. Szuławska A, Czyz M. Molecular mechanisms of anthracyclines action Postepy Hig Med Dosw (Online). 2006;60:78-100.

37. Blum JL, Dieras V, Lo Russo PM, et al. Multicenter, Phase II study of capecitabine in taxane $\square$ pretreated metastatic breast carcinoma patients. Cancer. 2001;92(7):1759-1768.

38. Weber BL, Vogel $\mathrm{C}$, Jones $\mathrm{S}$, et al. Intravenous vinorelbine as firstline and second-line therapy in advanced breast cancer. J Clin Oncol. 1995;13(11):2722-2730.

39. Romero Acuña L, Langhi M, Pérez J, et al. Vinorelbine and paclitaxel as first-line chemotherapy in metastatic breast cancer. J Clin Oncol. 1999; 17(1):74-74.

40. Burstein HJ, Kuter I, Campos SM, et al. Clinical activity of trastuzumab and vinorelbine in women with HER2-overexpressing metastatic breast cancer. J Clin Oncol. 2001;19(10):2722-2730.
41. Slamon DJ, Clark GM, Wong SG, et al. Human breast cancer: correlation of relapse and survival with amplification of the HER-2/neu oncogene. Science. 1987;235(4785):177-182.

42. Romond EH, Perez EA, Bryant J, et al. Trastuzumab plus adjuvant chemotherapy for operable HER2-positive breast cancer. $N$ Engl J Med. 2005;353(16):1673-1684.

43. Vu T, Claret FX. Trastuzumab: updated mechanisms of action and resistance in breast cancer. Front Oncol. 2012;2:62.

44. Baselga J, Carbonell X, Caslaneda Soto N, et al. Phase II study of efficacy, safety, and pharmacokinetics of trastuzumab monotherapy administered on a 3-weekly schedule. J Clin Oncol. 2004;23(10):2162-2171.

45. Pegram MD, Konecny GE, O Callaghan C, et al. Rational combinations of trastuzumab with chemotherapeutic drugs used in the treatment of breast cancer. J Natl Cancer Inst. 2004;96(10):739-749.

46. O Shaughnessy JA, Vukelja S, Marsland T, et al. Phase II study of trastuzumab plus gemcitabine in chemotherapy-pretreated patients with metastatic breast cancer. Clin Breast Cancer. 2004;5(2):142-147.

47. Rusnak DW, Lackey K, Affleck K, et al. The effects of the novel, reversible epidermal growth factor receptor/ErbB-2 tyrosine kinase inhibitor, GW2016, on the growth of human normal and tumor-derived cell lines in vitro and in vivo. Mol Cancer Ther. 2001;1(2):85-94.

48. Xia W, Mullin RJ, Keith BR, et al. Anti-tumor activity of GW572016: a dual tyrosine kinase inhibitor blocks EGF activation of EGFR/erbB2 and downstream Erk1/2 and AKT pathways. Oncogene. 2002;21(41):62556263.

49. Chu I, Blackwell $\mathrm{K}$, Chen $\mathrm{S}$, et al. The dual ErbB1/ErbB2 inhibitor, lapatinib (GW572016), cooperates with tamoxifen to inhibit both cell proliferation-and estrogen-dependent gene expression in antiestrogenresistant breast cancer. Cancer Res. 2005;65(1):18-25.

50. Geyer CE, Forster J, Lindquist D, et al. Lapatinib plus capecitabine for HER2-positive advanced breast cancer. N Engl J Med. 2006;355(26):27332743.

51. Toi M, Iwata H, Fujiwara Y, et al. Lapatinib monotherapy in patients with relapsed, advanced, or metastatic breast cancer: efficacy, safety, and biomarker results from Japanese patients phase II studies. $\mathrm{Br} J$ Cancer. 2009;101(10):1676-1682.

52. Blackwell KL, Burstein HJ, Storniolo AM, et al. Randomized study of Lapatinib alone or in combination with trastuzumab in women with ErbB2-positive, trastuzumab-refractory metastatic breast cancer. J Clin Oncol. 2010;28(7):1124-1130. 